\title{
The Detection of Spontaneous Venous Pulsation with Smartphone Video Ophthalmoscopy
}

This article was published in the following Dove Press journal:

Clinical Ophthalmology

\author{
Charlotte Laurent $\mathbb{D}^{1,2}$ \\ Sheng Chiong Hong (D) $^{3,4}$ \\ Kirsten R Cheyne $\mathbb{D}^{2}$ \\ Kelechi C Ogbuehi ${ }^{2}$ \\ 'Ophthalmology Department, Southern \\ District Health Board, Dunedin, New \\ Zealand; ' ${ }^{2}$ University of Otago, Dunedin, \\ New Zealand; ${ }^{3}$ Ophthalmology \\ Department, Mid Central District Health \\ Board, Palmerston North, New Zealand; \\ ${ }^{4}$ oDocs Eye Care Limited, Auckland, \\ New Zealand
}

Purpose: Spontaneous venous pulsation (SVP) has a high negative predictive value for raised intracranial pressure and is a useful sign when assessing patients with headache. The objective was to determine if smartphone-based video ophthalmoscopy can detect SVP.

Patients and Methods: In total 233 patients and 291 eyes were recruited from the Dunedin Hospital eye clinic from July to November 2018. Patients were examined by a clinician and graded for SVP with a slit lamp and 78 Dioptre lens. Videos were taken with a smartphone ophthalmoscope and graded by two separate clinicians blinded to the slit lamp findings.

Results: Only 272 eyes of 215 patients were included, as others failed in the inclusion criteria for overall video quality. Sensitivity was calculated as how likely the presence of SVP on video was indicative of the presence of SVP on slit lamp. Sensitivity was $84.77 \%$ for Observer 1, with 128 videos graded as positive for SVP on video ophthalmoscopy of the 151 identified as positive on slit lamp examination. Sensitivity was $76.82 \%$ for Observer 2 with 116 videos correctly identified. The false positive rate was calculated as the number of videos graded positive for SVP that had been graded as negative on slit lamp examination. This was $10.74 \%$ for observer 1 and $31.40 \%$ for observer 2 .

Conclusion: This study demonstrates that SVP is detected by video ophthalmoscopy. This may be a useful triage, telemedicine and referral tool to be used for patients with headache in a primary care setting.

Keywords: technology, telemedicine, neurology, headache
Correspondence: Charlotte Laurent Ophthalmology Department, Dunedin Public Hospital, 201 Great King Street, Dunedin 9016, New Zealand Tel +642 2l 2654347

Email charlotteclairelaurent@gmail.com

\section{Introduction}

Headache is a common complaint in primary care; it accounts for $4.4 \%$ of primary care consultations and $0.5-0.8 \%$ of emergency department presentations in the United Kingdom. ${ }^{1-3}$ These patients often present a diagnostic challenge as the majority of headaches are due to benign primary headache disorders. ${ }^{1,2}$ However, in a small proportion of cases the headache may represent a serious underlying pathology. ${ }^{1,2}$ Raised intracranial pressure (ICP) is one such pathology which may present with headache, it can be challenging to diagnose as neuroimaging is often normal and accurate ways to assess ICP are highly invasive. ${ }^{2,4}$ The most accurate way to assess ICP is with an intraventricular catheter, however in the emergency department lumbar puncture is the gold standard for the diagnosis of raised ICP. 1,2,4 Due to the invasive nature of these tests, it is essential that patients undergo a thorough assessment to establish who is at risk of having raised ICP, and thus who requires further invasive testing. ${ }^{1,2,4}$ The assessment of spontaneous venous pulsation (SVP) is a useful clinical sign which may aid the clinician's risk assessment of patients with headache. ${ }^{4}$ 
SVP is a dynamic clinical sign in which the pulsation of the retinal veins occurs with the cardiac cycle. ${ }^{4-8}$ It is most commonly appreciated as the retinal veins cross the optic disc, it may be obvious or subtle and limited to a portion of a single vessel. ${ }^{4-8}$ The underlying mechanism for this phenomenon is not fully understood, however it has been demonstrated that venous collapse occurs due to the difference in pulse pressure between the intracranial space and the intraocular space. ${ }^{4-10}$ This pressure difference is transmitted across the lamina cribrosa and across the vessels at the optic disc. ${ }^{8,9}$ This causes pulsation of the retinal veins rather than the retinal arteries due to their compliant thin walls. ${ }^{8,9}$

The presence of SVP is highly sensitive for the patient not having raised intracranial pressure. ${ }^{4-10}$ This is due to the fact that when patients have raised ICP, the translaminar pressure gradient which causes SVP is reduced causing the cessation of SVP. ${ }^{8}$ There is some debate about the sensitivity of the presence of SVP for normal ICP. ${ }^{8}$ Early studies conducted suggest that if SVP is present, the ICP is less than $190 \mathrm{mmH}_{2} \mathrm{O}$ at the time of examination, implying $100 \%$ sensitivity. ${ }^{5,6}$ A more recent study demonstrated that the sensitivity of the presence of SVP for a normal intracranial pressure was 0.89 , however patients in this study were examined with un-dilated pupils. ${ }^{8}$ The absence of SVP is not specific for raised ICP as the incidence of SVP is highly variable and may be present in up to $90 \%$ of the normal population. ${ }^{5-8}$ This relationship between SVP and ICP means that the assessment of SVP can act as an indirect qualitative assessment of ICP. ${ }^{4}$ As such it is highly useful as part of an assessment of patients with headaches, in part because it is non-invasive and not traumatic for patients. ${ }^{4}$

Despite this, examining the fundus and detecting SVP can be a challenge for clinicians. ${ }^{11-14} \mathrm{SVP}$ is best detected through a dilated pupil and using appropriate magnification such as with a direct ophthalmoscope or slit lamp with a 78D or $60 \mathrm{D}$ lens. ${ }^{5,15}$ It is also essential to view the optic disc for an appropriate length of time to accurately assess for SVP. ${ }^{15}$ The direct ophthalmoscope is portable, widely available and has the advantage of providing appropriate magnification in order to see SVP. ${ }^{5,11}$ Despite this clinicians and medical students have reported they are not comfortable performing direct ophthalmoscopy and are not confident in reporting their findings or identifying abnormalities. ${ }^{11-14}$ Direct ophthalmoscopy takes skill to perform and requires practice, however there is very little time spent learning ophthalmology in medical schools around the world. ${ }^{11-14,16,17}$
Furthermore, time is a significant barrier for clinicians to practice and perform this skill in busy clinics. ${ }^{11}$

Multiple alternatives for fundus examination have been developed including smart phone ophthalmoscopes. ${ }^{15,17,18}$ These consist of a lens adapter attached to a smart phone camera. ${ }^{14,17,18}$ Many studies have suggested that smart phone ophthalmoscopy may be easier to teach in medical school and is preferred by medical students. ${ }^{14,17,18}$ Improving access to smartphone ophthalmoscopy removes the barrier of developing the skill required to perform direct ophthalmoscopy. ${ }^{11,18}$ This may also enable teaching to focus on interpreting the findings of fundoscopy instead. ${ }^{11}$ Smart phone fundoscopy also has the ability to store images to monitor change and provides the opportunity for images to be stored for remote specialist review. ${ }^{17,18}$ Smart phones are common amongst medical professionals and smartphone ophthalmoscopy technology is becoming cheaper and more accessible. ${ }^{14,17,18}$ This means it may soon be available in the primary care setting. ${ }^{14,17,18} \mathrm{SVP}$ is a dynamic clinical sign and thus video ophthalmoscopy would be the most appropriate way for clinicians to identify it.

This study aimed to validate smart phone-based video ophthalmoscopy for the detection of SVP. We sought to determine the reliability of a smartphone-based video ophthalmoscope, compared with slit lamp fundus biomicroscopy, for the detection of SVP.

\section{Methods}

This was a prospective study in which an unselected population of adult patients were recruited from the Dunedin Hospital eye clinic. Patients presenting to the tertiary hospital outpatient eye department who were pharmacologically dilated for examination from July 2018 through November 2018 were eligible for recruitment. Patients with media opacities such as corneal scars and cataracts were excluded from the study. Formal scientific consultation was obtained, and ethical approval was granted through The University of Otago Human Ethics Committee (Health) approval number H15/071. Consultation with the indigenous Māori people of New Zealand was done through Ngāi Tahu. Informed consent was obtained prior to the examination in accordance with the tenets of the Helsinki Declaration. Patients were graded for SVP using the slit-lamp biomicroscope and a 78 Dioptre lens by an experienced ophthalmology registrar. Eyes were graded individually as being positive for SVP or negative for SVP. 
Videos were taken using the oDocs nun ophthalmoscope with an iPhone 8 (Apple Inc, CA, USA) mobile phone. The oDocs nun is an indirect ophthalmoscope with a built-in inverting prism designed to operate like a Welch Allyn PanOptic ophthalmoscope. Videos were taken at 1080 p at 30 frames per second. Figure 1 demonstrates the device in use. The person in Figure 1 has provided written informed consent for their image to be published.

Two clinicians blind to the slit lamp results, independently observed the videos taken with the smartphone ophthalmoscope and were asked to determine whether or not SVP was present in each video. SVP was only graded as present if the observers were certain it was there, to reflect how this tool would need to be used in a clinical setting. Videos would need to be graded as certainly present or uncertain in order to avoid missing cases where SVP was not present, as these patients may be at risk of raised intracranial pressure. SVP was graded as not identified when it was not seen or if there was any uncertainty. These responses were then compared to the slit lamp gradings and interobserver agreement was determined. Figure 2 demonstrates an example of the videos taken.

\section{Results}

Over the study period, 233 patients were recruited, and 291 eyes were examined. Videos of poor quality or where the optic disc was not captured were excluded leaving 272 eyes of 215 patients included in the study. This was composed of 128 right eyes and 144 left eyes. The average age of the patients in the study group was 64.5 years $(\mathrm{SD}=$ 12.5) as shown in Table 1.

Using the slit lamp biomicroscope, one hundred and fiftyone (151) out of 272 eyes (55.5\%) were graded as positive for SVP. These ratings were regarded as the gold standard, against which the observers' ratings were compared.

The results of the two observers are shown in Table 2, along with the gold standard results from slit lamp examinations. Of the 151 eyes identified as positive for SVP using the slit lamp, Observer 1 correctly identified 128 as positive for SVP on video ophthalmoscopy and Observer 2 correctly identified 116 . Sensitivity was calculated as how likely the presence of SVP on video was indicative of the presence of SVP on slit lamp. For Observer 1 the sensitivity was $84.77 \%$ (CI 78.03-90.09), and for Observer 2 it was $76.82 \%$ (CI 69.27-83.29). Positive predictive value which is the proportion of patients identified with SVP present on video ophthalmoscopy with SVP present on slit

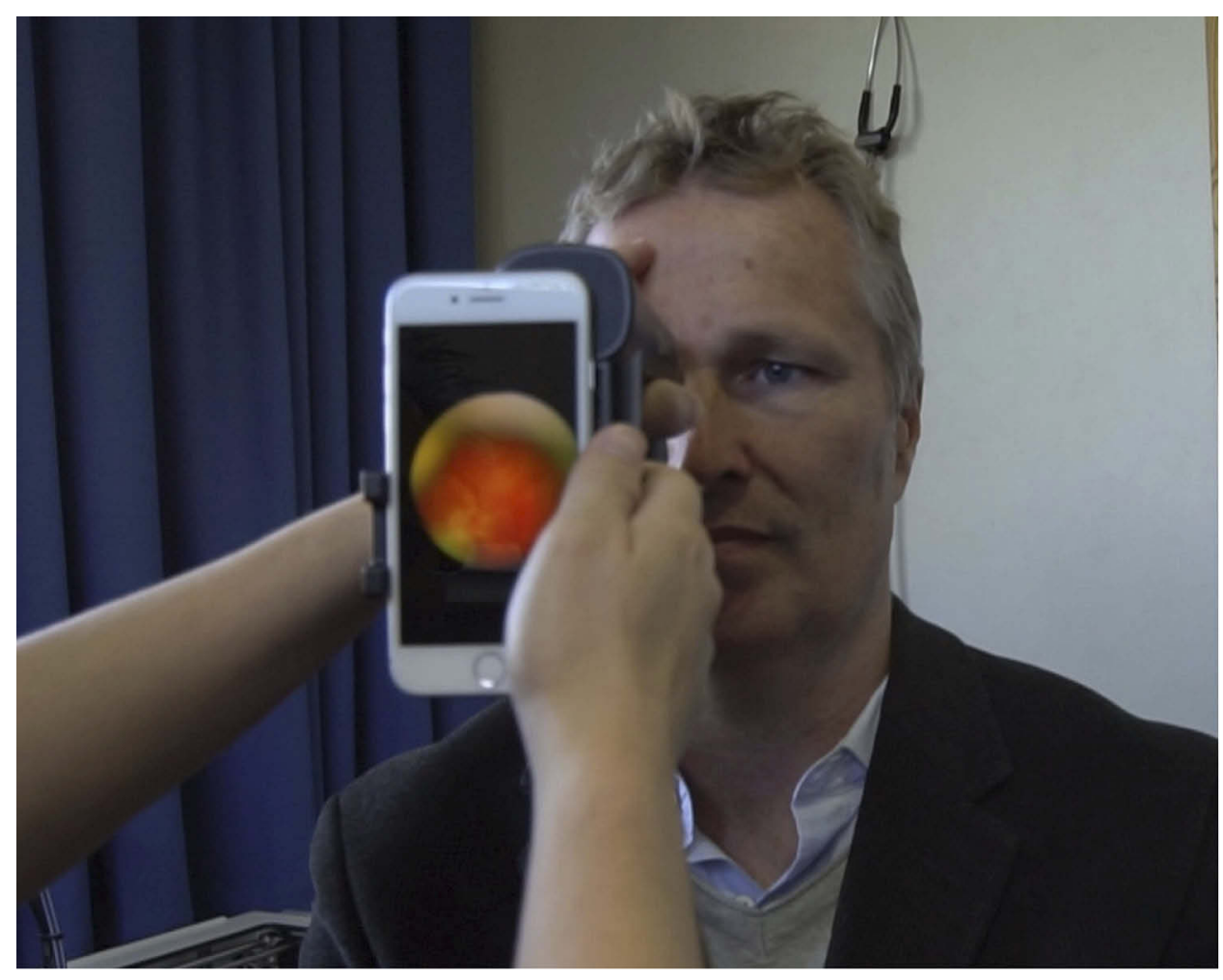

Figure I Demonstration of the oDocs nun ophthalmoscope in use. 

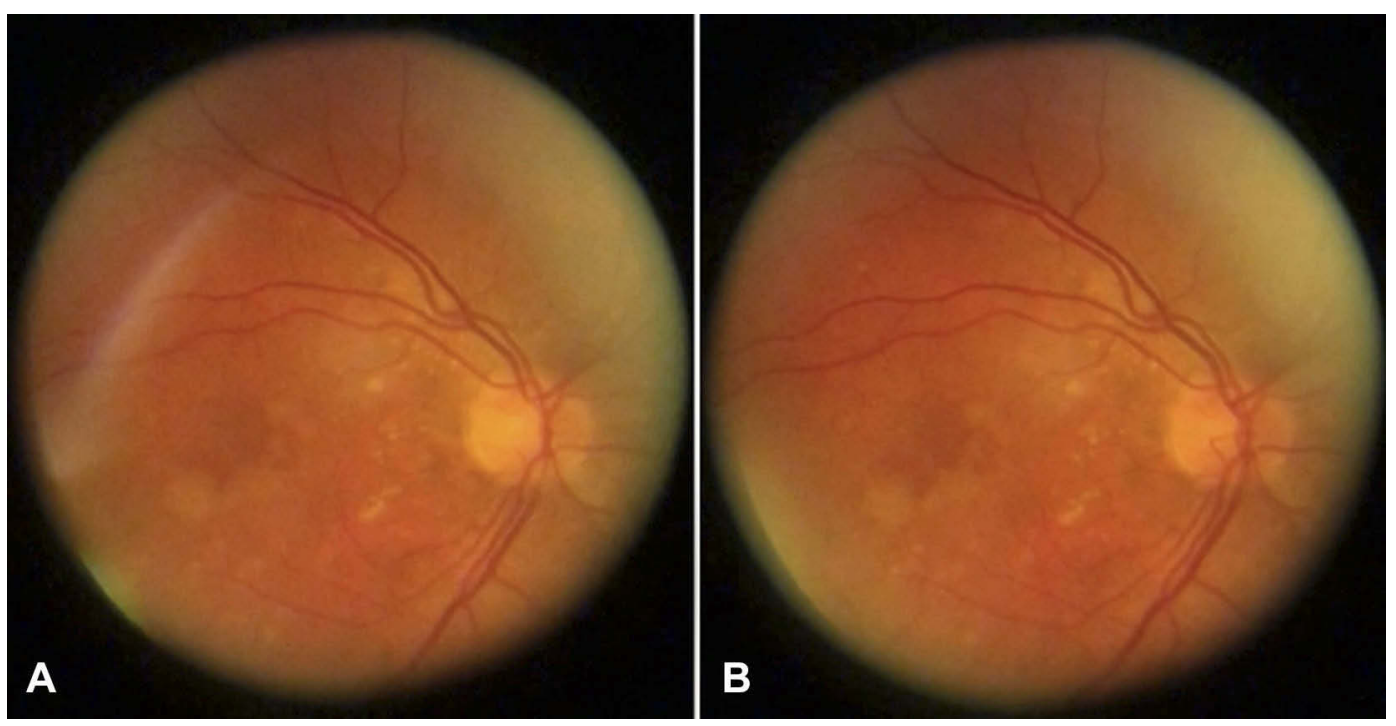

Figure 2 Still images taken from the same video showing SVP. (A) Shows collapse of the veins. (B) Shows dilation of the veins. SVP is seen at the optic disc.

lamp was 90.78\% (CI 85.43-94.30) for Observer 1 and $75.32 \%$ (CI 69.27-83.29) for Observer 2. Of the videos graded positive for SVP the number graded as negative for SVP on slit lamp examination was 13 for Observer 1 and 38 for Observer 2 giving a false positive rate of $10.74 \%$ and $31.40 \%$ respectively.

Of the 121 videos graded as negative on the slit lamp examination, 108 were correctly identified on video as negative by Observer 1 and 83 were correctly identified as negative by Observer 2. Specificity, which is the probability that SVP would be absent on video when it was absent on slit lamp examination was $89.26 \%$ (CI 82.33-

Table I Patient Demographics

\begin{tabular}{|l|l|}
\hline Average age & $64.5(\mathrm{SD}=12.5)$ \\
Age range & $24-92$ \\
Number of right eyes & 128 \\
Number of left eyes & 144 \\
\hline
\end{tabular}

Table 2 Responses Given by Two Blind Observers Asked to Identify SVP on Videos, Compared with Gold Standard Identification Using Slit Lamp

\begin{tabular}{|l|l|l|l|l|}
\hline \multirow{2}{*}{} & \multicolumn{2}{|l|}{ SVP Identified on Video } & \multicolumn{2}{l|}{$\begin{array}{l}\text { SVP Not Identified on } \\
\text { Video }\end{array}$} \\
\cline { 2 - 5 } & Observer I & Observer 2 & Observer I & Observer 2 \\
\hline $\begin{array}{l}\text { SVP present } \\
\text { on slit lamp }\end{array}$ & 128 & 116 & 23 & 35 \\
\hline $\begin{array}{l}\text { SVP not } \\
\text { present on } \\
\text { slit lamp }\end{array}$ & 13 & 38 & 108 & 83 \\
\hline
\end{tabular}

94.15) for Observer 1 and 68.60\% (CI 59.53-79.73) for Observer 2. These results are displayed in Table 3.

The interobserver reliability between observer 1 and observer 2 was calculated using the kappa co-efficient and was moderate to substantial $(\mathrm{kappa}=0.608)$. Percentage agreement was $80.5 \%$.

Age groups were also analysed individually. A histogram demonstrating the frequency of distribution is shown in Figure 3. Age groups with small numbers of participants were not analysed. Sensitivity and specificity were calculated for the 7 major age groups, demonstrated in Table 4. The sensitivity for observer 2 was lower in the age group (42-47) which is likely due to the small number of patients in this age group who had SVP on slit lamp examination. Overall, the results for individual age groups were consistent with the study results as a whole, with observer 1 having a higher sensitivity and specificity across the age groups.

\section{Discussion}

Our study was designed to test if smartphone video ophthalmoscopy is reliable for detecting SVP. Sensitivity

Table 3 Results of Two Observers for the Identification of SVP on Video Being Present on Slit Lamp Examination

\begin{tabular}{|l|l|l|}
\hline Results & Observer I (95\% Cl) & Observer $\mathbf{2}$ (95\% Cl) \\
\hline Sensitivity & $84.77 \%(78.03-90.09)$ & $76.82 \%(69.27-83.29)$ \\
Specificity & $89.26 \%(82.33-94.15)$ & $68.60 \%(59.53-76.73)$ \\
PPV & $90.78 \%(85.43-94.30)$ & $75.32 \%(69.8 I-80.12)$ \\
NPV & $82.44 \%(76.23-87.30)$ & $70.34 \%(63.39-76.46)$ \\
Accuracy & $86.76 \%(82.15-90.56)$ & $73.16 \%(67.48-78.33)$ \\
\hline
\end{tabular}




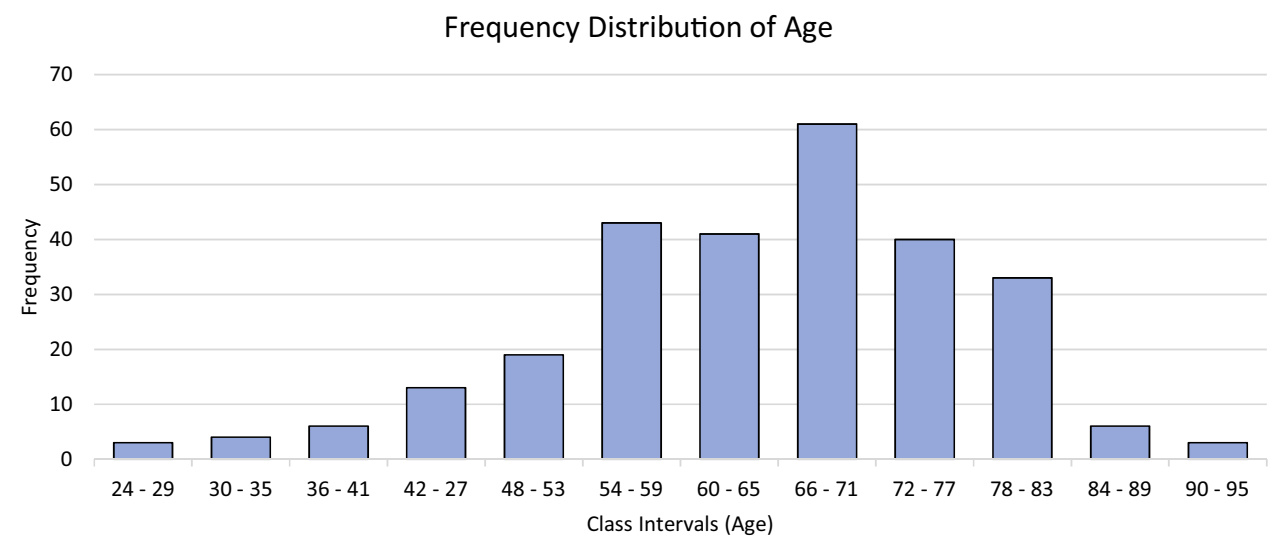

Figure 3 Graph Demonstrating the Frequency of Distribution of Age.

was $84.77 \%$ and $76.82 \%$ for the two observers respectively. This shows that video ophthalmoscopy is fairly sensitive for the detection of SVP if it is present on slit lamp examination. Patients were examined for SVP in a routine manner using the slit lamp biomicroscope and a 78 Dioptre lens and while this is the most appropriate way to look for SVP, clinical examination is not infallible.

Specificity is the probability that SVP would be absent on video when it was absent on slit lamp examination and was $89.26 \%$ and $68.60 \%$ respectively. The disparity between observers may be because videos were graded as negative if there was any uncertainty. As a result, the specificity values reported in this study are not a valid reflection of the tools ability to detect the absence of SVP. Clinically it is safer to grade patients with any uncertainty as SVP absent, to avoid missing cases where patients may be at risk for raised intracranial pressure.

Both observers had a number of false positive results where videos were graded as positive for SVP when slit lamp examination graded them as negative. This means patients without SVP could potentially be missed by the

Table 4 Sensitivity and Specificity for Observer I and Observer 2 Based on Age Groups

\begin{tabular}{|l|l|l|l|l|}
\hline \multirow{2}{*}{$\begin{array}{l}\text { Age } \\
\text { Group }\end{array}$} & \multicolumn{2}{|l|}{ Observer I } & \multicolumn{2}{l|}{ Observer 2 } \\
\cline { 2 - 5 } & Sensitivity & Specificity & Sensitivity & Specificity \\
\hline $42-47$ & $66.7 \%$ & $90.0 \%$ & $33.3 \%$ & $70.0 \%$ \\
$48-53$ & $85.7 \%$ & $100 \%$ & $85.7 \%$ & $75.0 \%$ \\
$54-59$ & $76.0 \%$ & $83.3 \%$ & $64.0 \%$ & $77.8 \%$ \\
$60-65$ & $100.0 \%$ & $100.0 \%$ & $76.5 \%$ & $70.8 \%$ \\
$66-71$ & $84.6 \%$ & $90.9 \%$ & $79.5 \%$ & $54.5 \%$ \\
$72-77$ & $89.7 \%$ & $63.6 \%$ & $82.8 \%$ & $54.5 \%$ \\
$78-83$ & $80.0 \%$ & $84.6 \%$ & $75.0 \%$ & $76.9 \%$ \\
\hline
\end{tabular}

triage tool. Alternatively, this demonstrates the difficulties faced with grading new technology against subjective human observation as the gold standard. It is possible that videos in which SVP was marked as present may have been missed on slit lamp examination.

This technology may be used as a platform for artificial intelligence and the automatic detection of SVP. This is an area of technology that is continuously evolving. Current applications of the automatic detection of dynamic clinical signs include the smart phone pupillometer and optokinetic nystagmus detection. ${ }^{19-21}$ We trialed the current videos with the slit lamp gradings using an open source platform for deep learning. We used the deep learning framework Inception V3 by Tensorflow (Alphabet Inc, CA, USA). Our initial experiments were unsuccessful, and we were unable to train the artificial intelligence with the number and quality of videos available.

Our study has several limitations. Firstly, 6.5\% of videos were excluded prior to the study due to poor video quality. Camera shake made the detection of SVP more challenging and contributed to many of the videos that the observers were uncertain about. Therefore, before this technology can be utilized in the real world, several improvements need to be made. Firstly, by taking videos in higher definition we could improve the overall video quality and probably the accuracy of interpretation. Mounting the smartphone ophthalmoscope may stabilize the videos, reduce error and ease the detection of SVP.

Other limitations pertain to the population selected for the trial. Patients were recruited from a tertiary hospital eye clinic rather than general practice, and there was no correlation made between patients ICP and the presence of SVP. This may not reflect the population intended to be examined by the tool. Patients with media opacity were 
also excluded, this may be a confounder as videos taken of these patients will be of poor quality therefore they would not be suitable for examination with this tool. In clinical practice poor quality videos would need to be disregarded.

Other study limitations include the relatively small number of participants recruited and grading the videos with only two observers. Further studies need to be undertaken with more patients and observers. We would aim to increase the number of observers grading SVP using the slit lamp as well as the number of individuals grading the videos. Increasing the number of patients in the study would also improve the reliability and would improve our ability to train artificial intelligence to detect SVP. The use of a subjective gold standard is also a limitation. Utilizing optical coherence tomography to detect SVP sub-clinically may be valuable as an objective gold standard against which to compare both the smartphone ophthalmoscope videos and slit-lamp findings. Patients with confirmed raised intracranial pressure would also need to be examined and included in the study to see if this could be used as a reliable screening tool.

\section{Conclusion}

In conclusion, video ophthalmoscopy can successfully detect SVP. While video ophthalmoscopy will not replace the conventional slit lamp examination it may be a useful triage and referral tool to be used for patients with headache in a primary care or emergency department setting. The ability to store videos with this technology would allow for remote specialist review in teleconsultation which would improve access to care for patients in rural settings. This tool may be easier to use than the direct ophthalmoscope and could aid in the assessment of these patients with challenging presentations and help with triaging of referrals.

\section{Acknowledgments}

The authors would like to acknowledge the Dunedin Ophthalmology Department, Southern District Health Board of New Zealand. No funding was obtained for this study.

\section{Disclosure}

Dr. Hong was the director of oDocs Eye Care Limited, during the conduct of the study. The authors report no other conflicts of interest in this work.

\section{References}

1. Locker TE, Thompson C, Rylance J, Mason SM. The utility of clinical features in patients presenting with nontraumatic headache: an investigation of adult patients attending an emergency department. Headache. 2006;46(6):954-961. doi:10.1111/hed.2006.46.issue-6

2. Duncan $\mathrm{CW}$. Neuroimaging and other investigations in patients presenting with headache. Ann Indian Acad Neurol. 2012;15(Suppl 1): S23-S32. doi:10.4103/0972-2327.99995

3. Latinovic R, Gulliford M, Ridsdale L. Headache and migraine in primary care: consultation, prescription, and referral rates in a large population. J Neurol Neurosurg Psychiatry. 2006;77(3):385-387. doi:10.1136/jnnp.2005.073221

4. Beau B. State-of-the-art review: non-invasive assessment of cerebrospinal fluid pressure. J Neuroophthalmol. 2014;34(3):288-294. doi:10.1097/WNO.0000000000000153

5. Jacks AS, Miller NR. Spontaneous retinal venous pulsation: aetiology and significance. J Neurol Neurosurg Psychiatry. 2003;74(1):7-9. doi:10.1136/jnnp.74.1.7

6. Levin BE. The clinical significance of spontaneous pulsations of the retinal vein. Arch Neurol. 1978;35(1):37-40. doi:10.1001/archneur.1978.00500 250041009

7. Morgan WH, Lind CR, Kain S, Fatehee N, Bala A, Yu DY. Retinal vein pulsation is in phase with intracranial pressure and not intraocular pressure. Invest Ophthalmol Vis Sci. 2012;53(8):4676-4681. doi:10.1167/iovs.12-9837

8. Wong SH, White RP. The clinical validity of the spontaneous retinal venous pulsation. $J$ Neuroophthalmol. 2013;33(1):17-20. doi:10.10 97/WNO.0b013e3182622207

9. Morgan WH, Hazelton ML, Yu DY. Retinal venous pulsation: expanding our understanding and use of this enigmatic phenomenon. Prog Retin Eye Res. 2016;55:82-107. doi:10.1016/j. preteyeres.2016.06.003

10. Kain S, Morgan WH, Yu DY. New observations concerning the nature of central retinal vein pulsation. Br J Ophthalmol. 2010;94 (7):854-857. doi:10.1136/bjo.2009.169813

11. Mackay DD, Garza PS, Bruce BB, Newman NJ, Biousse V. The demise of direct ophthalmoscopy: A modern clinical challenge. Neurol Clin Pract. 2015;5(2):150-157. doi:10.1212/CPJ.000000000 0000115

12. van Velden JS, Cook C, Du Toit N, et al. Primary health eye care: evaluation of the competence of medical students in performing fundoscopy with the direct ophthalmoscope. S Afr Fam Pract. 2010;52:341-343.

13. Zhang HH, Hepschke JL, Shulruf B, et al. Sharpening the focus on ophthalmology teaching: perceptions of medical students and junior medical officers. Clin Exp Ophthalmol. 2018;46(9):984-993. doi:10.1111/ceo.2018.46.issue-9

14. Mamtora S, Sandinha MT, Ajith A, Song A, Steel DHW. Smart phone ophthalmoscopy: a potential replacement for the direct ophthalmoscope. Eye (Lond). 2018;32(11):1766-1771. doi:10.1038/ s41433-018-0177-1

15. Legler U, Jonas JB. Assessment of the spontaneous pulsations of the central retinal vein in daily ophthalmic practice. Clin Exp Ophthalmol. 2007;35(9):870-871. doi:10.1111/j.1442-9071.2007.01 641.x

16. Sim D, Hussain A, Tebbal A, Daly S, Pringle E, Ionides A. National survey of the management of eye emergencies in the accident and emergency departments by senior house officers: 10 years on-has anything changed? Emerg Med J. 2008;25(2):76-77. doi:10.1136/ emj.2007.049999

17. Wu AR, Fouzdar-Jain S, Suh DW. Comparison study of funduscopic examination using a smartphone-based digital ophthalmoscope and the direct ophthalmoscope. J Pediatr Ophthalmol Strabismus. 2018;55(3):201-206. doi:10.3928/01913913-20180220-01 
18. Muiesan ML, Salvetti M, Paini A, et al. Ocular fundus photography with a smartphone device in acute hypertension. J Hypertens. 2017;35(8):1660-1665. doi:10.1097/HJH.0000000000001354

19. Mariakakis A, Baudin J, Whitmire E, et al. PupilScreen: using smartphones to assess traumatic brain injury. Proc ACM Interact Mob Wearable Ubiquitous Technol. 2017;1(3):1-27. doi:10.1145/3139486
20. Kim T, Youn J. Development of a smartphone-based pupillometer. J Opt Soc Korea. 2013;17(3):249-254. doi:10.3807/JOSK.2013.17. 3.249

21. Sangi M, Thompson B, Turuwhenua J. An optokinetic nystagmus detection method for use with young children. IEEE $J$ Transl Eng Health Med. 2015;3:1600110. doi:10.1109/JTEHM.2015.2410286

\section{Publish your work in this journal}

Clinical Ophthalmology is an international, peer-reviewed journal covering all subspecialties within ophthalmology. Key topics include: Optometry; Visual science; Pharmacology and drug therapy in eye diseases; Basic Sciences; Primary and Secondary eye care; Patient Safety and Quality of Care Improvements. This journal is indexed on PubMed

Submit your manuscript here: https://www.dovepress.com/clinical-ophthalmology-journal
Central and CAS, and is the official journal of The Society of Clinical Ophthalmology (SCO). The manuscript management system is completely online and includes a very quick and fair peer-review system, which is all easy to use. Visit http://www.dovepress.com/ testimonials.php to read real quotes from published authors. 